\title{
Diffusion tensor imaging of occult injury of optic radiation following optic neuritis in multiple sclerosis
}

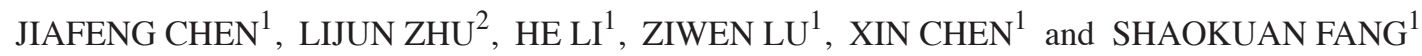 \\ ${ }^{1}$ Department of Neurology, Neuroscience Centre, The First Teaching Hospital of Jilin University, Changchun, Jilin 130012; \\ ${ }^{2}$ Department of Neurology, The Third Teaching Hospital of Jilin University, Changchun, Jilin 130021, P.R. China
}

Received May 29, 2015; Accepted July 15, 2016

DOI: $10.3892 /$ etm.2016.3635

\begin{abstract}
Multiple sclerosis (MS) is easily detected by routine magnetic resonance imaging (MRI). However, it is not possible to detect early or occult lesions in MS by routine MRI, and this may explain the inconsistency between the severity of the lesions found by MRI and the degree of clinical disability of patients with MS. The present study included 10 patients with relapsing-remitting MS and 10 healthy volunteers. Each patient underwent routine 3.0 T MRI, diffusion tensor imaging (DTI), and diffusion tensor tractography (DTT). Optic nerve and optic radiation were analyzed by DTI and DTT. The fractional anisotropy (FA), mean diffusivity (MD), $\lambda_{/ /}$, and $\lambda_{\perp}$ values were measured. In the 10 patients with MS, 7 optic nerves were affected, and 13 optic nerves were not affected. Cranial MRI showed that optic nerve thickening and hyperintensity occurred in 2 patients with MS. In the directionally encoded color maps, a hypointensive green signal in the optic nerve was observed in 3 patients with MS. The FA values were significantly lower and the $\mathrm{MD}, \lambda_{/ /}$, and $\lambda_{\perp}$ values were significantly higher in the affected and unaffected optic nerves and optic radiations in patients with MS in comparison with controls $(\mathrm{P}<0.05)$. There were no significant differences in these values between the affected and unaffected optic nerves and optic radiation in patients with MS $(\mathrm{P}>0.05)$. Diffusion tensor imaging is sensitive in the detection of occult injury of the optic nerve and optic radiation following optic neuritis. Diffusion tensor imaging may be a useful tool for the early diagnosis, treatment and management of MS.
\end{abstract}

\section{Introduction}

Multiple sclerosis (MS) is one of the most common demyelinating diseases of the central nervous system; the

Correspondence to: Professor Shaokuan Fang, Department of Neurology, Neuroscience Centre, The First Teaching Hospital of Jilin University, 71 Xinmin Street, Changchun, Jilin 130012, P.R. China

E-mail: fang20063536@sina.com

Key words: multiple sclerosis, diffusion tensor imaging, diffusion tensor tractography, optic nerve, optic radiation worldwide incidence of is increasing and is currently estimated to be $\sim 3.6 / 100,000$ individuals per year in women and 2.0/100,000 in men (1). It affects numerous brain regions, such as the corpus callosum, subcortical white matter, white matter surrounding the lateral ventricle, brain stem, cerebellum, spinal cord and optic nerves, and the course of MS can be relapsing-remitting or progressive (2). MS is the most common disabling neurological disease that affects young adults (3). MS is easily detected by routine magnetic resonance imaging (MRI) with a high detection rate; thus, MRI is a sensitive noninvasive diagnostic tool for MS (4). However, early or occult lesions in MS cannot be detected by routine MRI, and this may explain the inconsistency between the severity of the lesions found by MRI and the degree of clinical disability of patients with MS (5). Diffusion tensor imaging (DTI) has been used to detect occult injury of the corpus callosum in early MS (6). DTI may be a more sensitive imaging tool for the diagnosis of early or occult lesions in MS, compared with MRI (7).

DTI is a noninvasive imaging technique that detects white matter fiber bundles by measuring anisotropic water diffusion with an effective diffusion tensor (8). DTI can identify the lesion by presenting changes in color signals and directly showing the disruption and loosening of fibers; thus, it can be used for the evaluation of the severity of MS lesions (9). The optic radiation is a group of axons projecting from the lateral geniculate nucleus to the primary visual cortex, and is commonly affected by MS $(10,11)$. Clinical and animal studies have shown that a reduction in the eigenvalue $\lambda_{/ /}$(also known as $\lambda_{1}$ ) of the diffusion tensor, which reflects the water diffusion parallel to the axon, is associated with axonal injury. An increase in the eigenvalue $\lambda_{\perp}$ (the average of the eigenvalues $\lambda_{2}$ and $\lambda_{3}$ of the diffusion tensor), which reflects the water diffusion perpendicular to the axon, is associated with myelin injury and demyelination $(12,13)$. To date, few DTI studies on optic nerve damage in patients with MS or optic radiation damage after optic neuritis have been reported. Previous studies have used 1.5 T MRI $(14,15)$, and to the best of our knowledge, none have used 3.0 T MRI.

In the present study, 3.0 T MRI was used to investigate the DTI features of the optic nerve and optic radiation in 10 patients with MS and 10 healthy volunteers. The purpose of this study was to identify the characteristic DTI features of the optic nerve and optic radiation following optic neuritis in patients with MS. 


\section{Materials and methods}

Patient selection. The Medical Ethics Committee of the First Hospital of Jilin University (Changchun, China) approved the study, and all patients gave their informed consent prior to their inclusion in the study. This study included 10 patients (2 men and 8 women) with relapsing-remitting MS (RRMS) who were hospitalized at the Department of Neurology of the First Hospital of Jilin University. Of the 10 RRMS patients, MS predominantly affected the brain in 8 cases and the spinal cord in 2 cases. The mean age of the patients was 37.3 years (range, 27-55 years). Ten age- and gender-matched healthy volunteers who underwent cranial MRI at this hospital for a routine physical examination during the same period were included as controls. The inclusion criteria for the controls were: i) No history of neurological diseases or systemic diseases that affect the nervous system; ii) no neurological signs or symptoms; iii) no abnormality in MRI; and iv) right-handed subjects.

DTI examinations. MRI was performed with a TrioTim $3.0 \mathrm{~T}$ MR imaging system (Siemens AG, Munich, Germany) with a 12-channel phased-array head coil at a gradient strength of $45 \mathrm{mT} / \mathrm{m}$. Each subject underwent axial scans to obtain cranial MRI and DTI images. Axial and coronal scans were performed to obtain DTI images of the optic nerve. The optic nerve was scanned from the optic papilla to the intraorbital region (Fig. 1). Prior to the scan, the patient's head was fixed, and the patient was instructed to close their eyes and reduce eyeball movement. Routine T1-weighted (T1WI) MRI scans were performed as follows: Echo time $\left(\mathrm{T}_{\mathrm{E}}\right), 2.46 \mathrm{msec}$; repetition time $\left(\mathrm{T}_{\mathrm{R}}\right), 440 \mathrm{msec}$; matrix, 256x320; field of view (FOV), 220x220 mm²; slice thickness, $5 \mathrm{~mm}$; and inversion angle, $130^{\circ}$. The T2WI parameters were as follows: $\mathrm{T}_{\mathrm{E}}, 93 \mathrm{msec} ; \mathrm{T}_{\mathrm{R}}, 5,000 \mathrm{msec}$; matrix, 320x320; FOV, 220x220 $\mathrm{mm}^{2}$; slice thickness, $5 \mathrm{~mm}$; and interslice gap, $1.5 \mathrm{~mm}$. The DTI parameters were as follows: Matrix, $128 \times 128$; FOV, $230 \times 230 \mathrm{~mm}^{2}$; total sections, 16 consecutive sections; slice thickness, $2 \mathrm{~mm}$; diffusion gradient encoding in 64 directions; two diffusion gradient fields $(b=0$ and $\left.\mathrm{b}=1,000 \mathrm{sec} / \mathrm{mm}^{2}\right) ; \mathrm{T}_{\mathrm{E}}, 97 \mathrm{msec} ; \mathrm{T}_{\mathrm{R}}, 5,000 \mathrm{msec}$; and total imaging time, $342 \mathrm{sec}$.

Data and image processing. The original DTI data were processed on the 3.0 T MR imaging workstation to obtain directionally encoded color (DEC) images, black and white FA images, diffusion-weighted imaging images, and apparent diffusion coefficient images using the Neuro 3D software. The circular region of interest (ROI) with a diameter of $2.034 \mathrm{~mm}$ was manually selected. The ROI included the optic neuritis-affected optic nerve (designated the affected optic nerve), the optic radiation connected to the affected optic nerve (designated the affected optic radiation), unaffected optic nerve and unaffected optic radiation in patients with MS and the optic nerve and optic radiation in the controls. The fractional anisotropy (FA; a value between 0 and 1 that described the degree of diffusion directivity) and the mean diffusivity (MD; a mean value of the diffusion in three dimensions) were measured. The eigenvalue $\lambda_{/ /}$of the diffusion tensor and eigenvalue $\lambda_{\perp}$ were recorded.

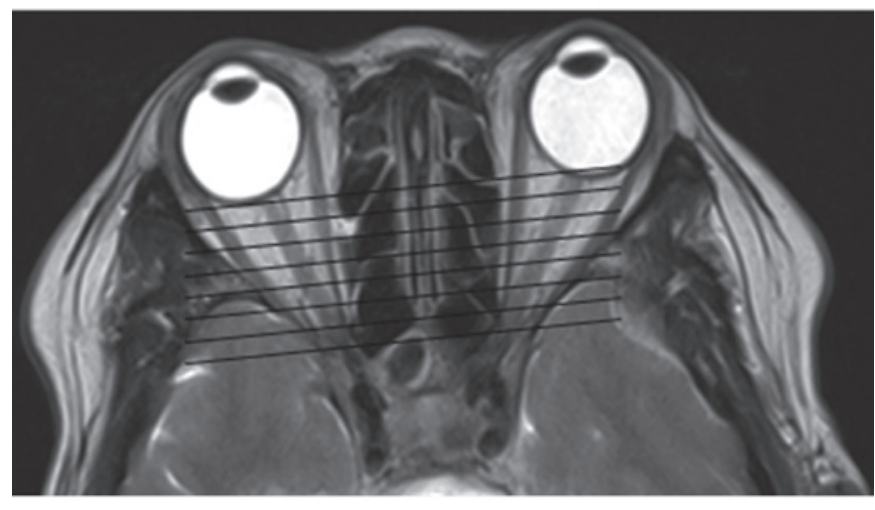

Figure 1. Magnetic resonance image of the optic nerve (scanned from the optic papilla to the intraorbital optic nerve).

Diffusion tensor tractography (DTT) was synthesized using tractography software, and reconstructed to obtain cranial 3D images based on the DEC maps. On the images, fibers of different directions were presented with different colors: Blue for the superior-inferior direction, green for the anterior-posterior direction, and red for the left-right direction. All ROIs were labeled based on their anatomy, and the direction of the fibers was automatically tracked. The morphology of the fibers, such as disruption and distortion, and the number of the fibers were directly observed.

Statistical analysis. Statistical analyses were performed using SPSS version 17.0 software (SPSS, Inc., Chicago, IL, USA). All data are presented as mean \pm standard deviation. Student's t-test or one-way analysis of variance was used to compare the difference between two or more than two groups, respectively. Levene tests or Welch tests were used to examine the homogeneity of variance. For data with homogeneous variance, least significant difference tests were used to compare differences. For data with inhomogeneous variance, Tamhane's T2 tests were used. $\mathrm{P}<0.05$ was considered to indicate a statistically significant difference.

\section{Results}

Optic nerves. Optic neuritis was defined as acute when the duration between disease onset and diagnosis was $>30$ days, and relapsing-remitting when the duration between disease onset and diagnosis was $>30$ days (16). All 10 patients with MS had relapsing-remitting optic neuritis. Of the 10 patients with MS, 7 optic nerves were affected and 13 optic nerves were not affected. Cranial MRI showed that optic nerve thickening and hyperintensity occurred in 2 cases (Fig. 2).

The data shown in Fig. 3 show the FA, MD, $\lambda_{/ /}$and $\lambda_{\perp}$ values of affected and unaffected optic nerves in patients with MS as well as those in controls. Compared with controls, the FA values of the patients with MS with affected and unaffected optic nerves were significantly lower $(\mathrm{P}<0.05$; Fig. 3$)$. In patients with MS, the MD, $\lambda_{/ /}$and $\lambda_{\perp}$ values of affected and unaffected optic nerves were significantly higher compared with those of controls ( $\mathrm{P}<0.05$; Fig. 3 ). There were no significant differences in the FA, MD, $\lambda_{/ /}$and $\lambda_{\perp}$ values between the affected and unaffected optic nerves in patients with MS ( $\mathrm{P}>0.05)$. 


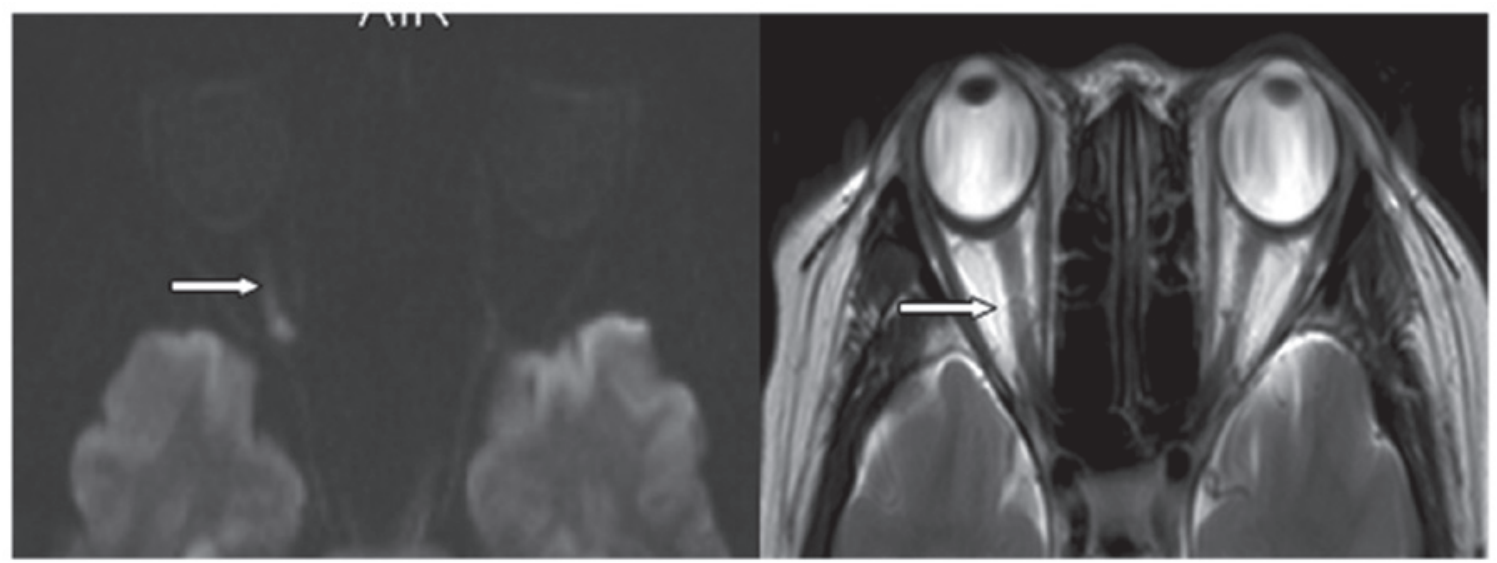

Figure 2. Cranial magnetic resonance images (left, diffusion-weighted; right T2-weighted) showing thickening and hyperintensity of the right optic nerve (arrows).

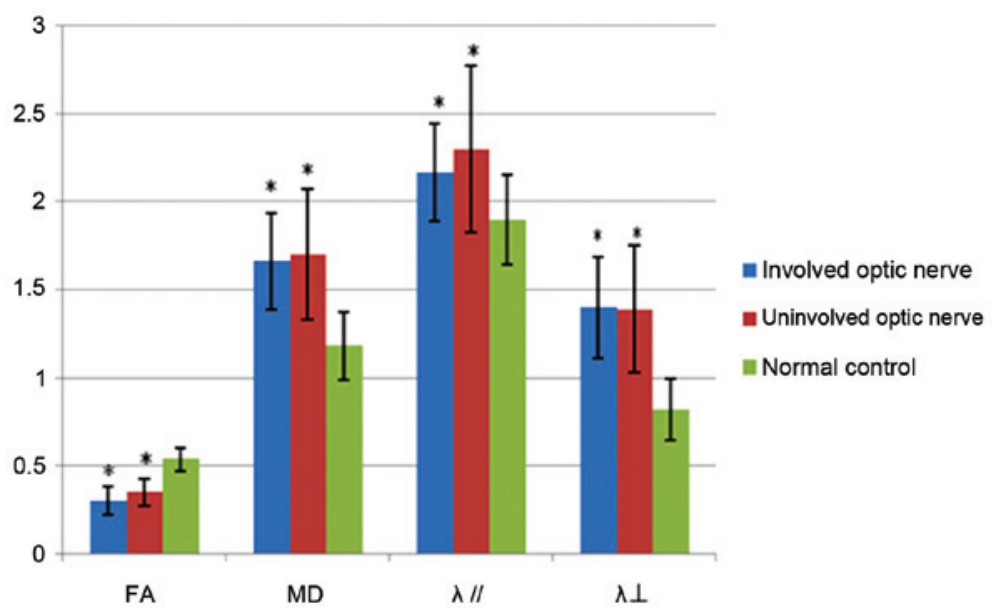

Figure 3. Fractional anisotropy (FA), mean diffusivity (MD), $\lambda_{\|}$, and $\lambda_{\perp}$ values of affected and unaffected optic nerves in patients with multiple sclerosis and controls. ${ }^{*} \mathrm{P}<0.05$ vs. controls.

The optic nerve in control cases exhibited hyperintensive signals in the FA map. In the DEC map, the optic nerve was green and clear with no evident artifacts or signal distortion. The signal intensity was almost the same as or slightly higher than that of the extraocular rectus. In the FA maps and DEC maps, the affected optic nerve displayed decreased intensity compared with the control (Fig. 4). A hypointensive green signal in the optic nerve was observed in 3 patients with MS.

Optic radiation. Seven optic nerves were connected to their affected optic radiation. MRI images showed no abnormality in affected optic radiation.

The FA, MD, $\lambda_{/ /}$and $\lambda_{\perp}$ values of the affected and unaffected optic radiations in patients with MS as well as those in controls are shown in Fig. 5. Compared with controls, the FA values of the affected and unaffected optic radiations were significantly lower in patients with MS ( $\mathrm{P}<0.05$; Fig. 5). The MD values of affected and unaffected optic nerves were significantly higher in patients with MS compared with controls ( $\mathrm{P}<0.05$; Fig. 5). There were no significant differences in the $\lambda_{/ /}$and $\lambda_{\perp}$ values between patients with MS and controls $(\mathrm{P}>0.05)$. Furthermore, there were no significant differences in the FA, MD, $\lambda_{/ /}$and $\lambda_{\perp}$ values between the affected and unaffected optic nerves in patients with MS $(\mathrm{P}>0.05)$.
In the FA map, the optic radiation was displayed as a high signal in controls. In the DEC map, the optic radiation that passed anteriorly to posteriorly between the tapetum corporis callosi and the afferent and efferent cortico-cortical fibers was manifested as a green signal. The green signal was easily distinguished from the blue signals of the tapetum corporis callosi and the afferent and efferent cortico-cortical fibers (Fig. 6A). The white high signal of the optic radiation in the FA map and the green signal of the optic radiation in the DEC map were not obviously altered in patients with MS compared with controls (Fig. 6B). Tractography of the optic chiasm showed that the number of fibers in the affected optic radiation was less than that in the contralateral unaffected optic radiation in patients with MS (Fig. 6B).

\section{Discussion}

Although routine MRI is a sensitive tool for detecting MS lesions, it poorly reflects the pathological nature of MS. In the present study, 3.0 T MRI was used to investigate DTI features of the optic nerve and optic radiation in 10 patients with MS in comparison with 10 healthy controls. It was found that in patients with MS compared with controls, the FA values were significantly lower for affected and unaffected 

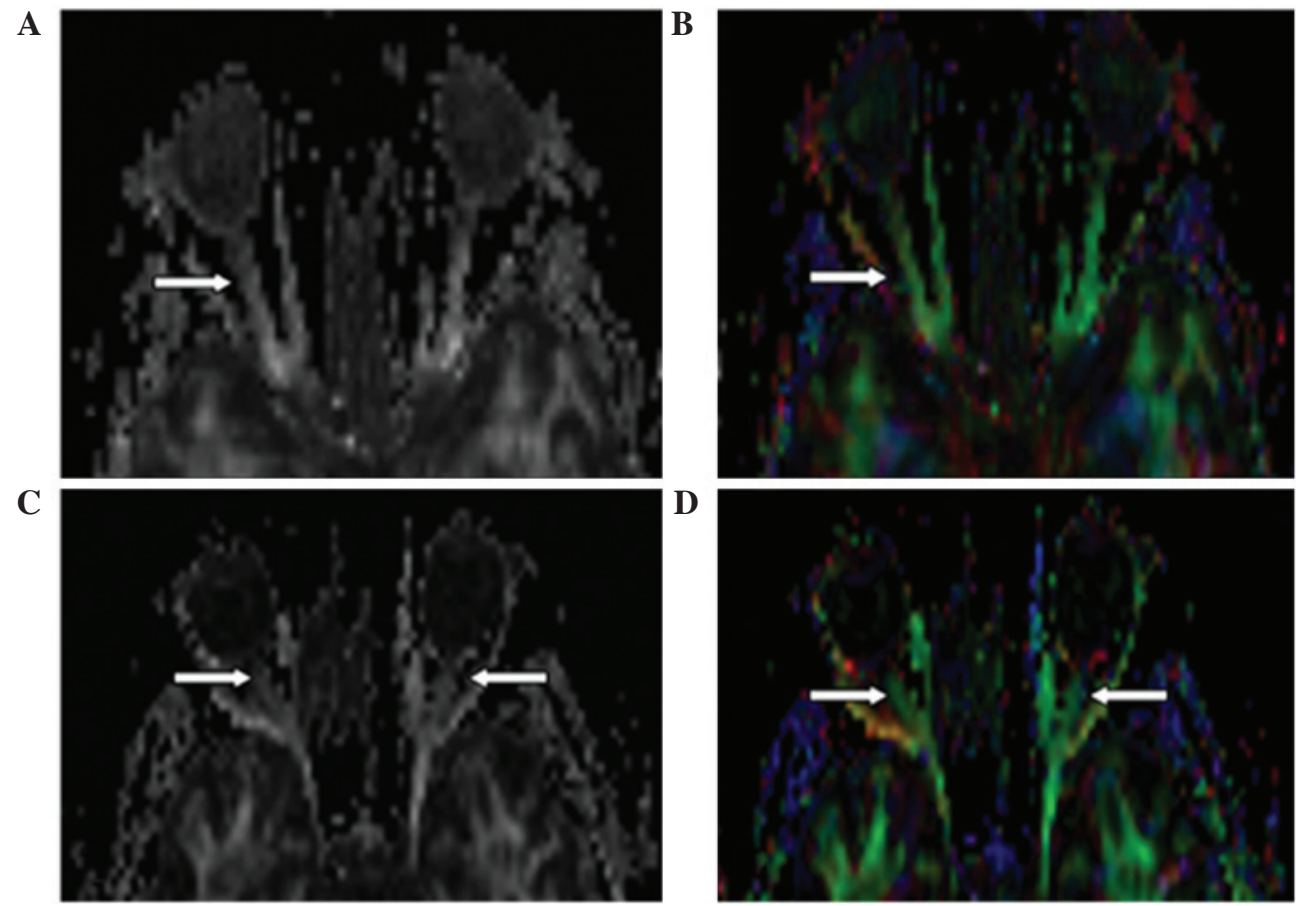

Figure 4.FA and DEC maps in two patients with multiple sclerosis. (A) FA and (B) DEC images show decreased intensity of the right optic nerve in a 48-year-old woman with right optic neuritis. (C) FA and (D) DEC images show decreased intensity of both optic nerves in a 31-year-old woman hospitalized due to numbness and weakness of the lower limbs for the past 10 days without visual impairment. Cranial magnetic resonance imaging showed no abnormality in the visual pathway. Visual evoked potential tests showed bilateral conduction blockade in the optic pathway. FA, fractional anisotropy; DEC, directionally encoded color.

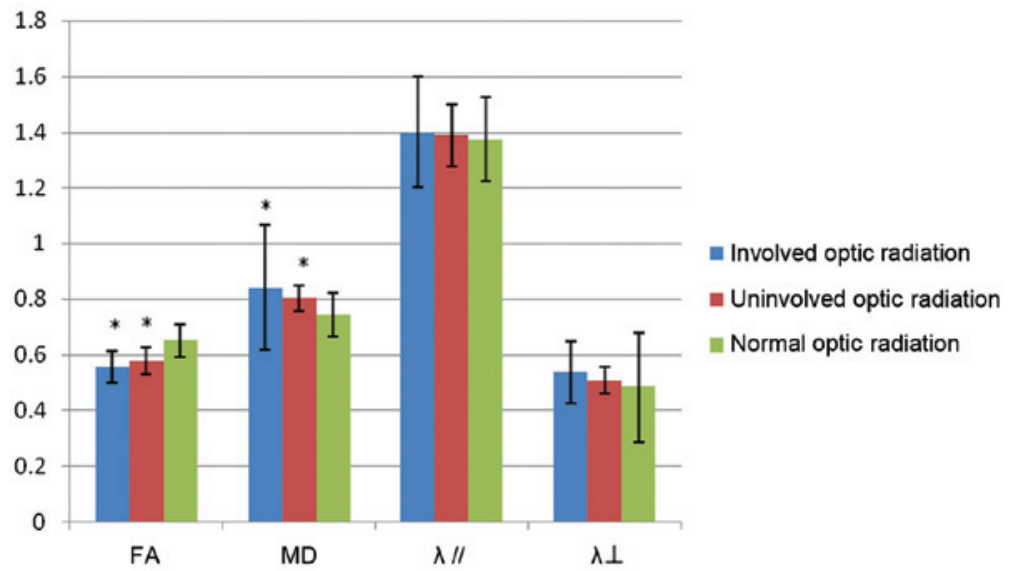

Figure 5. Fractional anisotropy (FA), mean diffusivity (MD), $\lambda_{\Perp}$, and $\lambda_{\perp}$ values of affected and unaffected optic radiations in patients with MS and in controls. ${ }^{*} \mathrm{P}<0.05$ vs. normal controls.

optic nerves and the $\mathrm{MD}, \lambda_{/ /}$, and $\lambda_{\perp}$ values were significantly higher. Furthermore, there were no significant differences in these values between the affected and unaffected optic nerves in patients with MS. Similar findings were reported by Kolbe et al (17).

The FA value in DTI is a sensitive measurement that reflects axonal integrity, and the MD value reflects water diffusion ability and velocity in the tissue. The findings of the present study that affected optic nerves exhibit a low FA value and a high MD value suggest that the affected optic nerves had decreased diffusion directivity and increased diffusivity. These observations suggest that the affected optic nerves exhibit axonal injury and demyelination. Hofling et al (18) reported that axonal density and demyelination are the main factors that affect water diffusion in the direction perpendicular to the optic nerve. Trip et al (19) found that compared with controls, the FA value was reduced and the MD value was increased in the affected and unaffected optic nerves in patients with a single unilateral episode of optic neuritis, and a reduction in the FA value and an increase in the MD value correlated with a reduction in the visual evoked potential amplitude, suggesting axonal loss in the affected and unaffected optic nerves.

In addition, the present study found that the optic nerve in patients with MS exhibited a lower $\lambda_{/ /}$value, which reflects water diffusion paralleling the axon and a lower $\lambda_{\perp}$ value, which reflects water diffusion perpendicular to the axon. A DTI study in a mouse model of traumatic axonal injury 

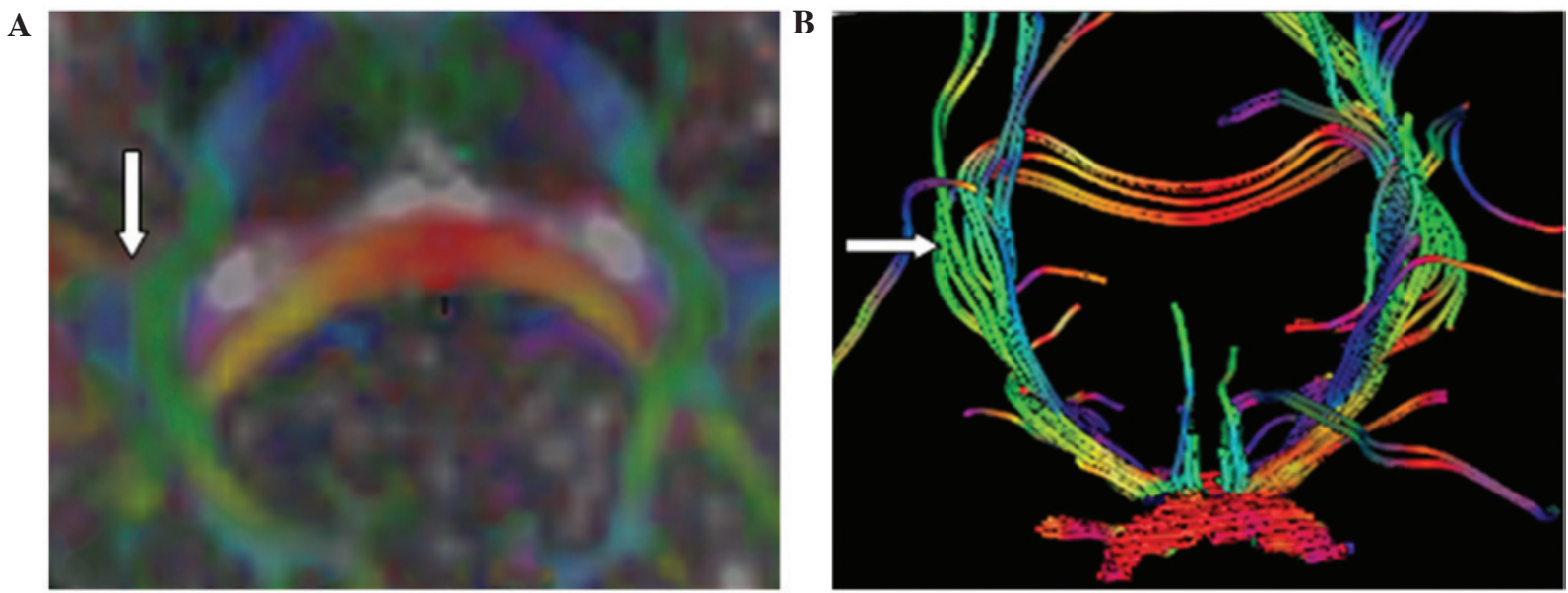

Figure 6. Directionally encoded color maps of optic radiation via (A) diffusion tensor imaging and (B) diffusion tensor tractography in a patient with multiple sclerosis (MS). (A) Optic radiation exhibited a green signal. (B) Tractography of the optic chiasm showed that the number of fibers in the affected optic radiation (right side) was less than the contralateral unaffected optic radiation (left side) in a 28-year-old woman with MS and right optic neuritis.

showed that the $\lambda_{/ /}$value was decreased $4 \mathrm{~h}$ after injury, recovered to normal 1 week after injury, and was significantly increased 1 month after injury, and these changes corresponded to demyelination, edema and persistent axonal injury (20).

In the present study, it was found that there were significant differences in the FA, MD, $\lambda_{\|}$and $\lambda_{\perp}$ values between patients with MS and controls, but no significant differences in these values were observed between the affected and unaffected optic nerves in patients with MS. This suggests that occult injury of unaffected optic nerves occurs before the patients experience visual impairment. A distant MS lesion or the presence of mild demyelination, axonal degeneration, necrosis and vacuolation may result in abnormalities in FA and MD values. These findings suggest that DTI is a useful technique for the early diagnosis and treatment of injury of the optic nerve.

In the present study, in the FA and DEC maps, it was observed that the affected optic nerve exhibited reduced signal intensity (Fig. 4A and B), and the unaffected optic nerve exhibited reduced signal intensity prior to the occurrence of visual impairment (Fig. 4C and D). Therefore, DTI parameters in combination with the findings in the FA map and DEC map are valuable for evaluating the injury severity and recovery of optic nerves, and are important for the early diagnosis of optic nerve injury.

There are several factors that may affect the quality of DTI images. First, during DTT, movement of the head or eye may lead to distortion of the optic nerve images. Secondly, artifacts may occur because the thin intraorbital optic nerve is wrapped by surrounding intraorbital fats and spinocerebral fluid. Thirdly, the image of the optic nerve can be distorted due to the air in the adjacent sinus, thus reducing tracking of the white matter fiber of the optic nerve (21). Fourthly, the diameter of the fiber, the density of glial cells, the tissue hydration status and myelination can affect the diffusivity of the optic nerve.

At the late stage of MS, brain atrophy and progressive neurological dysfunction can occur (22), but no new abnormal lesions are identified using routine MRI (23).
Kolbe et al (17) reported that the FA and $\lambda_{\|}$values were reduced, and the MD and $\lambda_{\perp}$ values were increased following optic neuritis in patients with MS, and suggested that anterograde trans-synaptic degeneration may occur following optic neuritis. In the present study, it was found that compared with controls, the FA values of the affected and unaffected optic radiations were significantly lower, and the MD value of affected and unaffected optic nerves were significantly higher in patients with MS, suggesting that trans-synaptic degeneration may occur in the affected and unaffected optic nerves.

In the present study, no significant difference in the FA, $\mathrm{MD}, \lambda_{/ /}$and $\lambda_{\perp}$ values were found between the affected and unaffected optic radiation. This may be due to unilateral optic injury affecting the contralateral side, since the optic nerves at each side are composed of fibers from both sides. It is also likely that the unaffected optic radiation may be associated with a mild and undetected occult lesion in the occipital cortex that may cause retrograde degeneration of unaffected optic radiation. These factors may contribute to the findings of a reduction in the FA value, and an increase in the MD, $\lambda_{/ /}$and $\lambda_{\perp}$ values of bilateral optic radiation. Currently, there have been few DTI studies on neurodegeneration of the optic radiation, and the mechanisms underlying the occurrence and progression of optic radiation degeneration remain unclear (24). The present study suggests that trans-synaptic degeneration may occur following optic neuritis. Tractography demonstrated that the number of fibers in the affected optic radiation was less than that in the contralateral unaffected optic radiation, and this further supports the possible presence of trans-synaptic degeneration. This may be due to chronic demyelination and axonal injury following optic neuritis inducing a reduction in afferent signals into the lateral geniculate nucleus and subsequently leading to atrophy of the optic radiation.

Therefore, DTI is indicated to be a sensitive tool for detecting optic radiation degeneration, and appears to be a suitable technique for use in the diagnosis of trans-synaptic degeneration of the optic radiation following chronic optic neuritis. 


\section{References}

1. Alonso A and Hernán MA: Temporal trends in the incidence of multiple sclerosis: A systematic review. Neurology 71: 129-135, 2008.

2. Al-Afasy HH, Al-Obaidan MA, Al-Ansari YA, Al-Yatama SA, Al-Rukaibi MS, Makki NI, Suresh A and Akhtar S: Risk factors for multiple sclerosis in Kuwait: A population-based case-control study. Neuroepidemiology 40: 30-35, 2013.

3. Berer K and Krishnamoorthy G: Microbial view of central nervous system autoimmunity. FEBS Lett 588: 4207-4213, 2014.

4. Simon JH: MRI outcomes in the diagnosis and disease course of multiple sclerosis. Handb Clin Neurol 122: 405-425, 2014.

5. Barkhof F: The clinico-radiological paradox in multiple sclerosis revisited. Curr Opin Neurol 15: 239-245, 2002.

6. Ge Y, Law M, Johnson G, Herbert J, Babb JS, Mannon LJ and Grossman RI: Preferential occult injury of corpus callosum in multiple sclerosis measured by diffusion tensor imaging. J Magn Reson Imaging 20: 1-7, 2004.

7. Gallo A, Rovaris M, Riva R, Ghezzi A, Benedetti B, Martinelli V, Falini A, Comi G and Filippi M: Diffusion-tensor magnetic resonance imaging detects normal-appearing white matter damage unrelated to short-term disease activity in patients at the earliest clinical stage of multiple sclerosis. Arch Neurol 62 803-808, 2005.

8. Gratsias G, Kapsalaki E, Kogia S, Dardiotis E, Tsimourtou V, Lavdas E, Kousi E, Pelekanou A, Hadjigeorgiou GM and Fezoulidis I: A quantitative evaluation of damage in normal appearing white matter in patients with multiple sclerosis using diffusion tensor MR imaging at $3 \mathrm{~T}$. Acta Neurol Belg 115: 111-116, 2015.

9. Beaulieu C: The basis of anisotropic water diffusion in the nervous system - a technical review. NMR Biomed 15: 435-455, 2002.

10. Reich DS, Smith SA, Gordon-Lipkin EM, Ozturk A, Caffo BS, Balcer LJ and Calabresi PA: Damage to the optic radiation in multiple sclerosis is associated with retinal injury and visual disability. Arch Neurol 66: 998-1006, 2009.

11. Sinnecker T, Oberwahrenbrock T, Metz I, Zimmermann H, Pfueller CF, Harms L, Ruprecht K, Ramien C, Hahn K, Brück W, et al: Optic radiation damage in multiple sclerosis is associated with visual dysfunction and retinal thinning - an ultrahigh-field MR pilot study. Eur Radiol 25: 122-131, 2015.

12. Klawiter EC, Xu J, Naismith RT, Benzinger TL, Shimony JS, Lancia S, Snyder AZ, Trinkaus K, Song SK and Cross AH: Increased radial diffusivity in spinal cord lesions in neuromyelitis optica compared with multiple sclerosis. Mult Scler 18: 1259-1268, 2012.
13. Song SK, Sun SW, Ju WK, Lin SJ, Cross AH and Neufeld AH: Diffusion tensor imaging detects and differentiates axon and myelin degeneration in mouse optic nerve after retinal ischemia. Neuroimage 20: 1714-1722, 2003.

14. Klistorner A, Sriram P, Vootakuru N, Wang C, Barnett MH, Garrick R, Parratt J, Levin N, Raz N, Van der Walt A, et al: Axonal loss of retinal neurons in multiple sclerosis associated with optic radiation lesions. Neurology 82: 2165-2172, 2014

15. Roosendaal SD, Geurts JJ, Vrenken H, Hulst HE, Cover KS, Castelijns JA, Pouwels PJ and Barkhof F: Regional DTI differences in multiple sclerosis patients. Neuroimage 44: 1397-1403, 2009.

16. Naismith RT, Xu J, Tutlam NT, Trinkaus K, Cross AH and Song SK: Radial diffusivity in remote optic neuritis discriminates visual outcomes. Neurology 74: 1702-1710, 2010.

17. Kolbe S, Bajraszewski C, Chapman C, Nguyen T, Mitchell P, Paine M, Butzkueven H, Johnston L, Kilpatrick T and Egan G: Diffusion tensor imaging of the optic radiations after optic neuritis. Hum Brain Mapp 33: 2047-2061, 2012

18. Hofling AA, Kim JH, Fantz CR, Sands MS and Song SK: Diffusion tensor imaging detects axonal injury and demyelination in the spinal cord and cranial nerves of a murine model of globoid cell leukodystrophy. NMR Biomed 22: 1100-1106, 2009.

19. Trip SA, Wheeler-Kingshott C, Jones SJ, Li WY, Barker GJ, Thompson AJ, Plant GT and Miller DH: Optic nerve diffusion tensor imaging in optic neuritis. Neuroimage 30: 498-505, 2006.

20. MacDonald CL, Dikranian K, Bayly P, Holtzman D and Brody D: Diffusion tensor imaging reliably detects experimental traumatic axonal injury and indicates approximate time of injury. J Neurosci 27: 11869-11876, 2007.

21. Smith SA, Williams ZR, Ratchford JN, Newsome SD, Farrell SK, Farrell JA, Gifford A, Miller NR, van Zijl PC, Calabresi PA and Reich DS: Diffusion tensor imaging of the optic nerve in multiple sclerosis: Association with retinal damage and visual disability. AJNR Am J Neuroradiol 32: 1662-1668, 2011.

22. Chard DT, Brex PA, Ciccarelli O, Griffin CM, Parker GJ, Dalton C, Altmann DR, Thompson AJ and Miller DH: The longitudinal relation between brain lesion load and atrophy in multiple sclerosis: A 14 year follow up study. J Neurol Neurosurg Psychiatry 74: 1551-1554, 2003.

23. Brex PA, Ciccarelli O, O'Riordan JI, Sailer M, Thompson AJ and Miller DH: A longitudinal study of abnormalities on MRI and disability from multiple sclerosis. N Engl J Med 346: 158-164, 2002.

24. Wieshmann UC, Symms MR, Clark CA, Lemieux L, Franconi F, Parker GJ, Barker GJ and Shorvon SD: Wallerian degeneration in the optic radiation after temporal lobectomy demonstrated in vivo with diffusion tensor imaging. Epilepsia 40: 1155-1158, 1999. 